\title{
Carbon nanotube enhanced shape memory polymer nanocomposites for development of biomedical devices
}

\begin{abstract}
This paper presented the synthesis and characterization of carbon nanotubes (CNTs) enhanced aliphatic urethane shape memory polymer (SMP) nanocomposites for the development of biomedical devices. Critical polymer properties, such as the glass transition temperature and shape memory function, have been tailored to desired applications, by adjusting the polymer composition. CNTs were uniformly dispersed within the polymer during nanocomposite fabrication. The synthesized SMPs nanocomposites were characterized to understand their thermal and mechanical properties. Novel biomedical devices for intracranial aneurysm treatment will be developed using these SMPs.
\end{abstract}

Keywords: Shape memory polymer, Carbon nanotubes, Nanocomposites, Aneurysm occlusion devices

\author{
Volume 6 Issue I - 2017 \\ Jingyu Wang,' Shoieb Chowdhury,' Dee Wu, ${ }^{2}$ \\ Bradley Bohnstedt, ${ }^{3}$ Yingtao Liu,' Chung-Hao \\ Lee' $^{1}$ \\ 'School of Aerospace and Mechanical Engineering, University of \\ Oklahoma, USA \\ 2Department of Radiological Sciences, University of Oklahoma \\ Health Sciences, USA \\ ${ }^{3}$ Department of Neurosurgery, University of Oklahoma Health \\ Sciences, USA
}

\section{Correspondence: Yingtao Liu, School of Aerospace and Mechanical Engineering, University of Oklahoma, Norman, OK, USA Email yingtao@ou.edu}

Received: July 05, 2017| Published: August 04, 2017

\section{Introduction}

Incidental rupture of an intracranial aneurysm results in subarachnoid hemorrhage, which accounts for about $5 \%$ of all strokes and causes about $10 \%$ of individual's death before reaching medical attention in the USA.$^{1}$ Endovascular embolization is a surgical procedure for treating the abnormal blood vessel by excluding the aneurysmal sac and neck from intracranial circulation by means of complete and lasting occlusion. Although it has become an established therapy in the past decade, current clinical challenges associated with disappointing long-term therapeutic outcomes, including high risk of aneurysm's rupture and recurrence rates (about $41 \%$ ), as well as incomplete occlusion due to the complex geometry of the treated aneurysms.$^{2,3}$

Shape memory polymers (SMPs) have attracted significant attention for their potential applications to develop novel biomedical actuators, sensors, devices and smart systems.$^{4}$ In general, SMPs possess the advantages of high elastic deformation, light-inweight, low cost, easy synthesis, and potential biocompatibility and biodegradability. Aneurysm occlusion devices are a promising application for biocompatible SMPs. Compared to current devices (Guglielmi detachable coil), SMP-based medical devices can reduce the risk of aneurysm rupture, increase the long-term treatment results, and be applicable to more complex aneurysm geometries. SMPs have been used to coat platinum coils in aneurysm treatment ${ }^{5}$ Promising initial results were obtained in in-vivo animal models. However, coil compaction and aneurysm recanalization rates increased due to resorption of the polymer coating, which resulted in low volumetric filling and need for retreatment. Recently, SMP foams based on DiAPLEX polyurethane have been investigated as a material to occlude aneurysms. Metcalfe et al. reported that the open cellular structure of SMP foams increased the growth of cells in neointima formation.$^{6}$ However, current SMP foams cannot be designed to specific geometry using patient specific aneurysm data.
In this paper, we reported our experimental efforts to synthesize and characterize aliphatic urethane SMPs and carbon nanotube (CNTs) reinforced nanocomposites for the development of novel devices for the endovascular embolization of intracranial aneurysms. We demonstrated that the critical polymer properties, such as the glass transition temperature, can be tailored by adjusting the polymer formula. The developed SMPs are the foundation for the development of medical devices with the optimal endovascular embolization capability for treating cerebral aneurysms.

\section{SMP synthesis and characterization}

Three monomers were used to synthesize the aliphatic urethane SMPs. All the materials were purchased from Sigma Aldrich and used without any additional modification. The monomers used were:

a. Hexamethylene diisocyanate (HDI)

b. N,N,N0,N0-tetrakis (hydroxypropyl) ethylenediamine (HPED)

c. Triethanolamine (TEA). Five different SMPs using different molar ratio of each monomer were prepared (HDI: HPED: TEA = 1.00:0.50:0.00, 1:00:0.45:0.067, 1.00:0.40:0.133, 1.00:0.35:0.20, 1.00:0.3:0.267 mole ratio). CNTs were added to increase the thermal conductivity of SMPs in this paper. An ultra-sonication based method was employed to uniformly disperse CNTs within the polymer. HPED and CNTs were first dispersed within a solvent before HDI and TEA were added to the solution. Once all the solvent has been evaporated, the mixed materials were cured in the designed molds for sample fabrication.

Dynamic mechanical analysis (DMA) was employed to determine the thermal transitions in the synthesized SMPs. Experiments were conducted using a Q800 dynamic mechanical analyzer (TA Instruments (New Castle, DE). Beam samples $(17.5 \times 15 \times 2 \mathrm{~mm})$ were tested using three-point bending mode from $50^{\circ} \mathrm{C}$ to $130^{\circ} \mathrm{C}$. All the synthesized SMPs were tested and repeated four times. In addition, cyclic tensile tests were carried out using dog bone samples (ASTM 
Standard D638-10, Type V) at temperature above their glass transition temperature to determine the suitability for device applications. The samples were tested in a dual-column Instron mechanical testing system (Instron, Norwood, MA) within an environmental chamber. The samples were clamped in the chamber and kept at the required temperature for 10 minutes before each test. Due to the low Young's modulus of such synthesized SMP materials, a load cell with the maximum load capacity of $100 \mathrm{~N}$ was used in the tests. Both pristine SMPs and CNTs reinforced SMPs were tested under cyclic tensile load conditions.

\section{Results and discussion}

The shape memory function of the synthesized SMPs nanocomposites were first investigated. Each straight beam sample was first immersed into hot water $\left(5-10^{\circ} \mathrm{C}\right.$ above the expected glass transition temperature), bended up to $90^{\circ}$, and cooled back to room temperature while maintaining the bended shape. Then the SMP nanocomposite sample was placed back to hot water and the material restored to the initial straight form autonomously. The samples at different shape is illustrated in Figure 1.
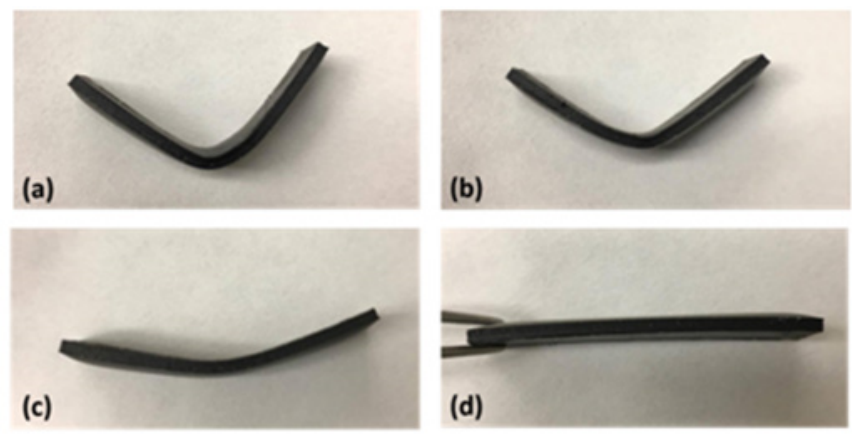

Figure I CNT reinforced SMP specimen changes shapes as heated above its glass transition temperature.

DMA tests were conducted to evaluate the glass transition temperature of different polymers with different molar ratio and polymer formulation. As we tailored the polymer formulation, the glass transition temperature of SMPs varied in the range of $65-80^{\circ} \mathrm{C}$. Since CNTs only increase the thermal and electrical conductivity of SMPs, the glass transition temperature of SMPs with dispersed CNTs are considered to the same as pure SMPs. CNT dispersion quality was examined using a field emission SEM system. The sample was sputter coated before SEM tests. Three SEM images with different magnifications were shown in Figure 2. It can be seen that CNTs were uniformly dispersed in the SMP matrix.
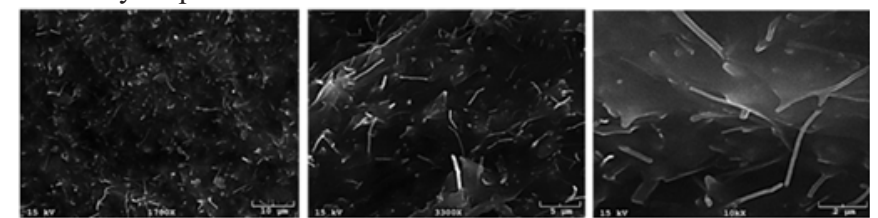

Figure 2 SEM images of different magnifications showing good CNT dispersion in SMP.

Cyclic tensile tests were used to evaluate the level of strain recovery for shape memory applications. The comparison of tensile results using pristine SMP and CNT reinforced SMP nanocomposites are shown in Figure 3. For the pristine SMP sample, it is noted that in the first cycle there was initial hysteresis, which can be due to the residual stress, material relaxation, or the re-arrangement of dangling chains and side-groups in the polymer. Once the SMP have been fully stretched and relaxed for the first cycle, the hysteresis became relatively insignificant. However, the initial hysteresis was not obvious in the CNT reinforced nanocomposite sample, mainly due to enhancement of mechanical properties by CNTs.

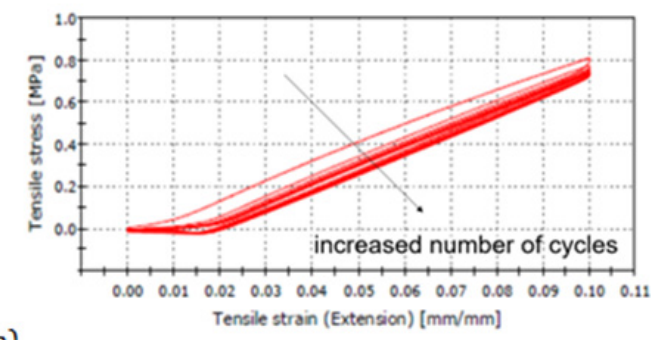

(a)

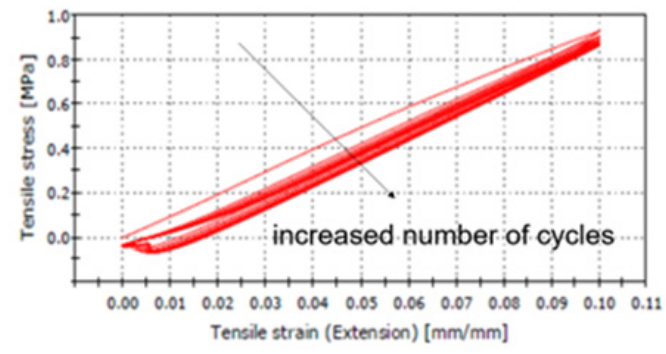

Figure 3 Cyclic tensile testing of CNT reinforced SMP sample up to 10\% engineering strain and 10 cycles, (a) sample without CNTs, (b) sample with CNTs.

\section{Conclusion}

This paper reported the development of nanocomposites using aliphatic urethane SMPs and CNTs for the potential application in the endovascular embolization of intracranial aneurysms. Our preliminary results showed that the synthesized polymer had good shape memory function when heated up to their glass transition temperature. The critical properties, such as their glass transition temperature, can be optimized by adjusting the polymer formulation. Moreover, cyclic mechanical tensile tests showed that the SMPs had initial hysteresis when operated above their glass transition temperature. The preliminary results showed good potential for the application of the developed SMP in the development of novel devices for aneurysm treatment.

\section{Acknowledgements}

The authors appreciate the financial support from the 2017 SEED Funding for Interdisciplinary Research program in the Gallogly College of Engineering at the University of Oklahoma, and the funding from the Oklahoma Shared Clinical and Translational Resources (NNIGMS U54GM104938). This research was supported in part by the grant awarded through the Faculty Investment Program from the Research Council of the University of Oklahoma Norman Campus.

\section{Conflicts of interest}

None.

\section{References}

1. Wermer MJ, van der Schaaf IC, Algra A et al. Risk of rupture of unruptured intracranial aneurysms in relation to patient and aneurysm characteristics. Stroke. 2007;38(4):1404-1410

2. Tsutsumi K, eki K, Morita A, Kirino T Risk of rupture from incidental cerebral aneurysms. Journal of neurosurgery. 2000;93(4):550-553. 
3. Winn HR, JA Jane, Taylor J et al. Prevalence of asymptomatic incidental aneurysms: review of 4568 arteriograms. Journal of neurosurgery. 2002;96(1):43-49.

4. Zhao Q, HJ Qi, Xie T Recent progress in shape memory polymer: new behavior, enabling materials, and mechanistic understanding. Progress in Polymer Science. 2015;49:79-120.
5. Murayama Y, Tateshima S, Gonzalez NR et al. Matrix and bioabsorbable polymeric coils accelerate healing of intracranial aneurysms. Stroke. 2003;34(8):2031-2037.

6. Metcalfe A, Desfaits AC, Salazkin I et al. Cold hibernated elastic memory foams for endovascular interventions. Biomaterials. $2003 ; 24(3): 491-497$. 